\title{
GREEN CLOUD COMPUTING SOLUTION FOR OPERATIONAL COST EFFICIENCY AND ENVIRONMENTAL IMPACT REDUCTION
}

\author{
Dr. V. Bindhu, \\ Professor and Head, Department of Electronics and Communication Engineering, \\ PPG Institute of Technology \\ Coimbatore, India. \\ Email id: vbindhuppg@gmail.com \\ Mr. C. Vijesh joe, \\ Assistant Professor, Department of Computer Science and Engineering, \\ VV College of Engineering, \\ Tirunelveli, India. \\ Email id:vijesh.joe@gmail.com
}

\begin{abstract}
Cloud computing provides information technology based services based on utilities to users across the globe. Application hosting for business, consumers and scientific domains is made possible with cloud computing. However, the data centres used for this purpose consume large amount of energy. This increases the operational cost and contribute carbon footprint affecting the environment. With increasing energy scarcities and global climate changes, it is essential to control power consumption. This paper presents a green cloud computing solution that addresses the issues of operational cost reduction and decreasing the carbon footprint and its impact on the environment. For this purpose we use data mining tools and auto-scaling with constraint satisfaction problems (CSP).
\end{abstract}

Keywords: Green cloud computing, environmental protection, operational cost,

\section{INTRODUCTION}

Cloud computing technology is a dynamic domain in information and communication technology. It is a distributed parallel computing technology that offers reliability, scalability, low cost and high performance. The factors such as improvement in energy efficacy, carbon footprint and e-waste lessening enables the development of green cloud computing technology. Large amount of servers and data centres work towards providing payper-use services to the consumers. These resources occupy huge area and require large amount of power.

There are mainly four types of cloud-computing architecture namely -

External or public architecture - Where an offsite provider maintains the resources on pay per-use basis. Internal or private architecture - A cloud infrastructure that works solely for a specific organization. 
Community architecture - The cloud is shared by multiple organizations with shared concerns.

Hybrid architecture - where public and private clouds may be integrated with third-party service providers.

The major concerns of cloud computing includes power management, energy consumption and efficiency, greenhouse gases $(\mathrm{GHG})$ and emission of $\mathrm{CO} 2$, virtualization of server and sustaining of natural resources. The concept of Green computing originated in 1987 with the basic idea of sustainable development. The primary focus of this development is to reduce energy consumption. Green computing involves efficient utilization of computers and related technologies with respect to environment.

Migration to virtual machines is an energy conscious technology that has been used in cloud computing environment in recent days. Nano Data Centres $(\mathrm{NaDa})$ provide a distributed computing platform and enables energy conservation of up to $30 \%$ compared to the traditional data centres [11]. In order to streamline the resource usage and enhance energy efficiency of the system task consolidation is performed. Hardware temperature control, server consolidation, compiler optimization, application software power optimization, dynamic energy consumption enabled operating system, virtual machine manager optimization, virtual machine live migration and network environment optimization are other notable energy efficient strategies that can be implemented in green cloud computing environment.

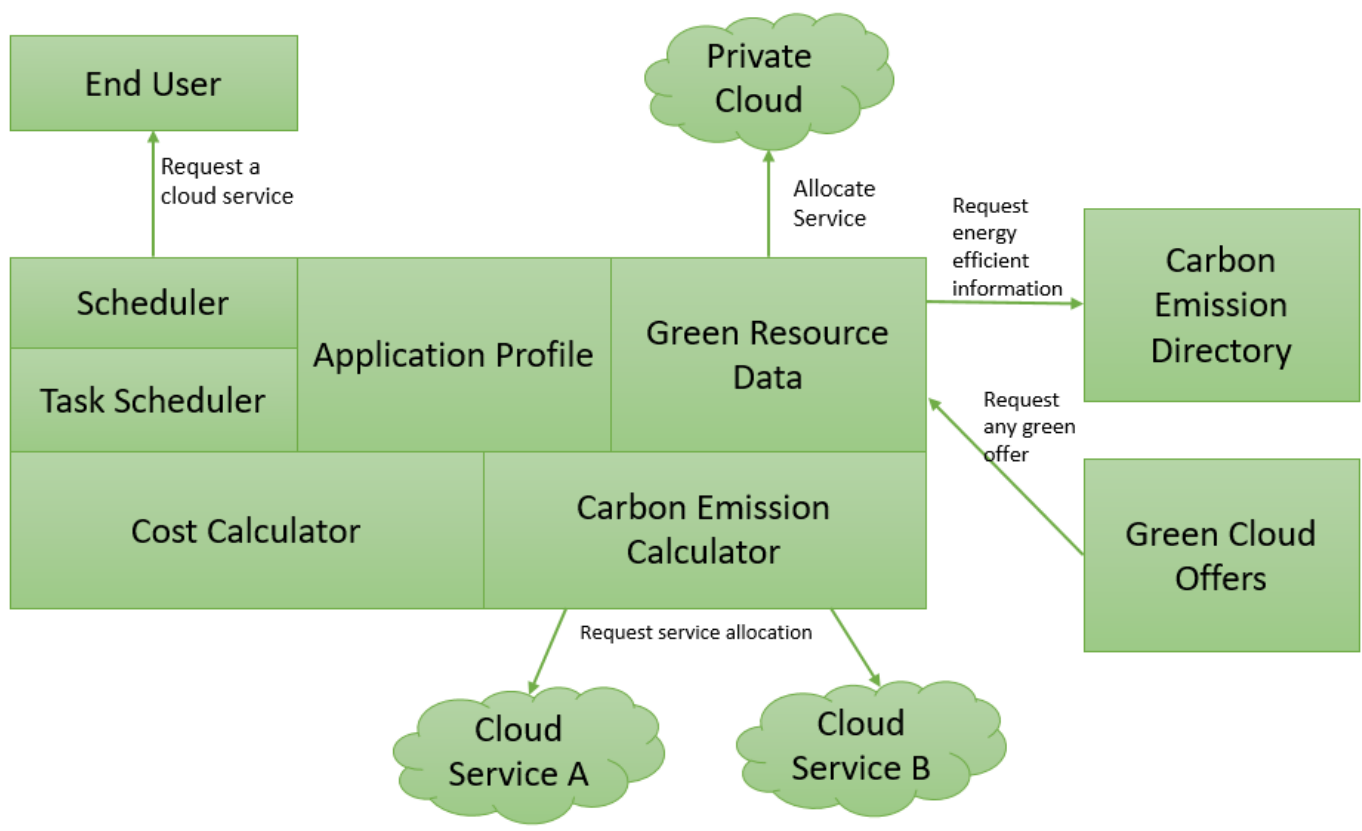

Figure 1 Green Cloud Computing Architecture

Figure 1 represents a simple Green Cloud Computing Architecture with the essential tools and technologies involved. Here cloud service A, B and the private cloud are the cloud data centres. These cloud data centres 
provide services such as Infrastructure as a Service (IaaS), Platform as a Service (PaaS) and Software as a Service (SaaS).

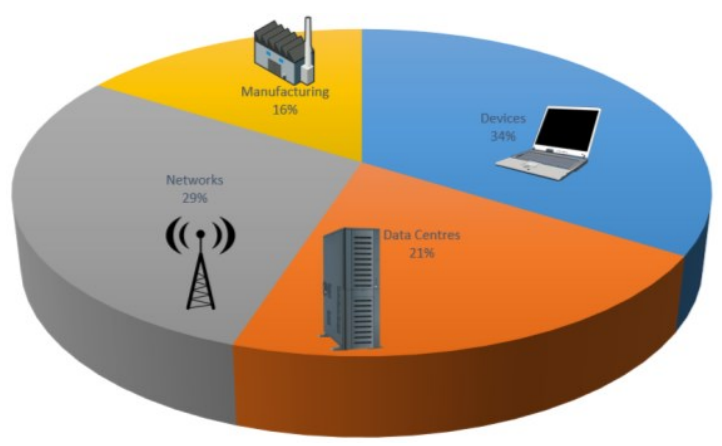

(a)

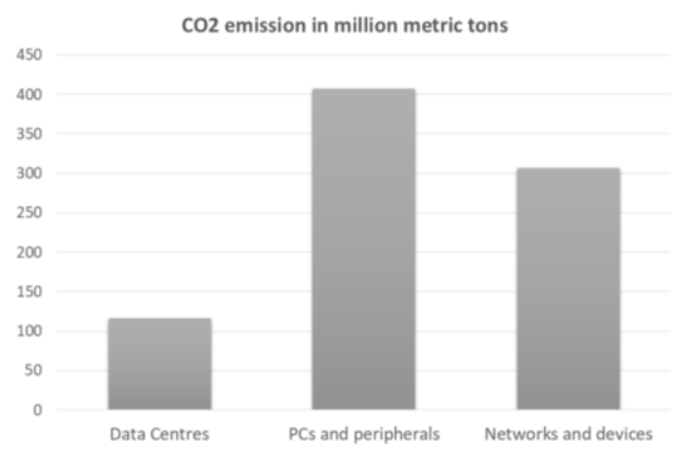

(b)

Figure 2 (a) Estimate of electricity consumption in IT sector and its components (2017) (b) CO2 emission in million metric tons.

Figure 2 (a) represents the average electricity consumption in IT sector in manufacturing sector, devices, networks and data centres based on data from Emerging Trends in Electricity Consumption for Consumer ICT. Figure 2 (b) represents the emission of $\mathrm{CO} 2$ in million metric tons from data centres, devices and networks.

\section{EXISTING LITERATURE}

Anubha Jain et al. [4] proposed several optimal ideas for green cloud computing. In order to moderate power consumption in a cloud computing environment, the major techniques that can be followed include 
reducing the CPU power dissipation, using advanced clock gating, using split plane power, using energyefficient processors, using renewable energy sources, using energy efficient storage and reducing cooling requirements. Kancharana et al. [12] analysed the advantages and drawbacks of green cloud computing. The peak hour utilization of this technology offers optimized power consumption. It also proves to be eco-friendly and reduces e-waste. Rapid technological changes and cost are a major glitch in the implementation of green cloud computing. But the disadvantages seem negligible when considering the advantages of the system.

Archana Patil et al. [13] performed a detailed study of the achievements and trends of green cloud computing. A cloud service broker module termed as the Green Cloud Provider (GCP) monitors the cloud infrastructure and its activities and endorses the related clouds as green. Maryam et al. [14] proposed a k-mean clustering based micro-genetic algorithm (KMGA) for dynamic consolidation and mapping task. This system consists of workload controller, local manager and global manager. This system reduced the virtual machine migration count and make-span when compared to hybrid techniques like genetic algorithm and particle swarm optimization.

Saurabh et al. [15] proposed a carbon aware green cloud framework that reduces the carbon footprint and improves the efficiency of the clouds. The paper also presented an IaaS cloud service provider based case study and a Carbon Efficient Green Policy (CEGP). Fumiko Satoh et al [16] developed an advanced cloud energy management system with optimized VM allocation tool and sensor management functions for reducing energy consumption of up to $30 \%$. Electricity bill minimization and reduction of carbon emission can be done by extending this system.

Mohammed et al. [17] suggested the usage of smart micro-grids for reducing energy cost and carbon emission in internet data centres (IDCs) along with energy efficiency for achieving green information and communication technology. This promotes cost efficiency, energy efficiency and reduction of carbon emission from the cloud data centre. Si-Yuan Jing et al. [18] proposed a feasible solution to certain major challenges in cloud computing such as quality of service, power consumption, security, standardization and software framework in IaaS system.

Rajkumar et al. [19] developed a dynamic resource allocation and provisioning algorithm taking into consideration, the synergy among several data centre infrastructural units including power units, hardware, software, and cooling. Piotr et al. [20] provided an outlook on manufacture, design, use and disposal of green cloud based technology.

http://irojournals.com/iroismac/ 


\section{PROPOSED WORK}

We use data mining and model driven engineering approaches for reduction of carbon footprint and improving the environment friendliness and optimizing the operational cost in green cloud computing environment.

\section{Environmental impact reduction}

In order to reduce the impact on environment, it is essential to analyse the carbon footprint and data centre utilization rate and hence promote green cloud computing. Data mining tools can be used for cloud computing decision support in data centres. In green cloud computing, the data centre achieves environmental friendliness by optimization of resources in such a way that pollution of atmosphere is reduces to the greatest extent thereby maintaining green surroundings. Data centres consume large amount of energy for powering as well as cooling of the servers. This produces an elevated carbon footprint. An organization's carbon footprint is the approximation of the total carbon dioxide let into the atmosphere that are basically generated by combustion of fossil fuels to supply power. Carbon footprint can be calculated using the formula

Electricity usage per yr in $\mathrm{kWh} \times$

Carbon footprint $=\frac{\text { CO2 national emission average in lbs per } \mathrm{kWh} \times 1 \text { metric ton }}{2204.6 \mathrm{lbs}}$

\section{Operational cost efficiency}

Auto-scaling feature can be applied to the cloud computing environment. This feature allows increasing the utilization of the server and reducing the idle time. This reduces the power consumption and hence reduce the emission on $\mathrm{CO} 2$ and creates a green computing environment. The configuration options and queue sizes increases exponentially and confounds attainment of minimal power consumption. We use a modeldriven engineering (MDE) approach and convert feature model representation into constraint satisfaction problems (CSP) and generate constrictions to guarantee maximum response time limit on auto-scaling.

In case of a feature selection problem, the CSP can be described as

$$
P_{F}=(F, C, \gamma)
$$


Where, $F$ represents the set of variables that describe the selection state of each feature. $\forall$ Feature $\mathrm{f}_{\mathrm{i}} \in \mathrm{F}, \mathrm{f}_{\mathrm{i}}=1$ if the feature is chosen in the derived configuration. $\mathrm{F}_{\mathrm{i}}=0$ if the $\mathrm{i}^{\text {th }}$ feature is not chosen. From the feature model, $\mathrm{C}$ captures the rules as limitations on the variables in F. $\gamma$ is an elective objective function that should be reduced or increased by the derived configuration.

\section{EVALUATION OF PERFORMANCE AND RESULT}

At any given time, the utilization $\mathrm{U}$ of a resource $\mathrm{r}_{\mathrm{i}}$ is given by

$$
U_{i}=\sum_{j=1}^{n} u_{i, j}
$$

Where $\mathrm{u}_{\mathrm{i}, \mathrm{j}}$ is the utilised resources by the task and $\mathrm{n}$ is the total number of task being executed at that time.

Figure 3 represents the comparison in performance based on energy consumption and $\mathrm{CO} 2$ emission between traditional method and proposed method of green cloud computing optimization. The analysis is done on a cloud computing data centre based on the utilization percentage of data transfer for a specific time limit. It is found that the proposed method offers optimal performance compared to the traditional cloud computing technique.

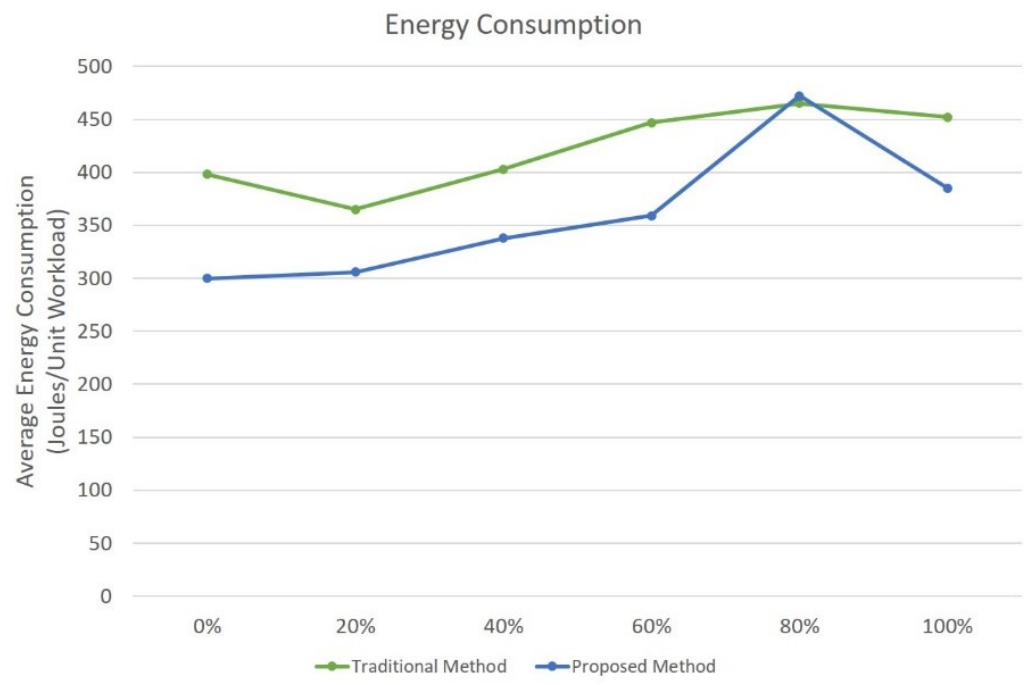

(a) 


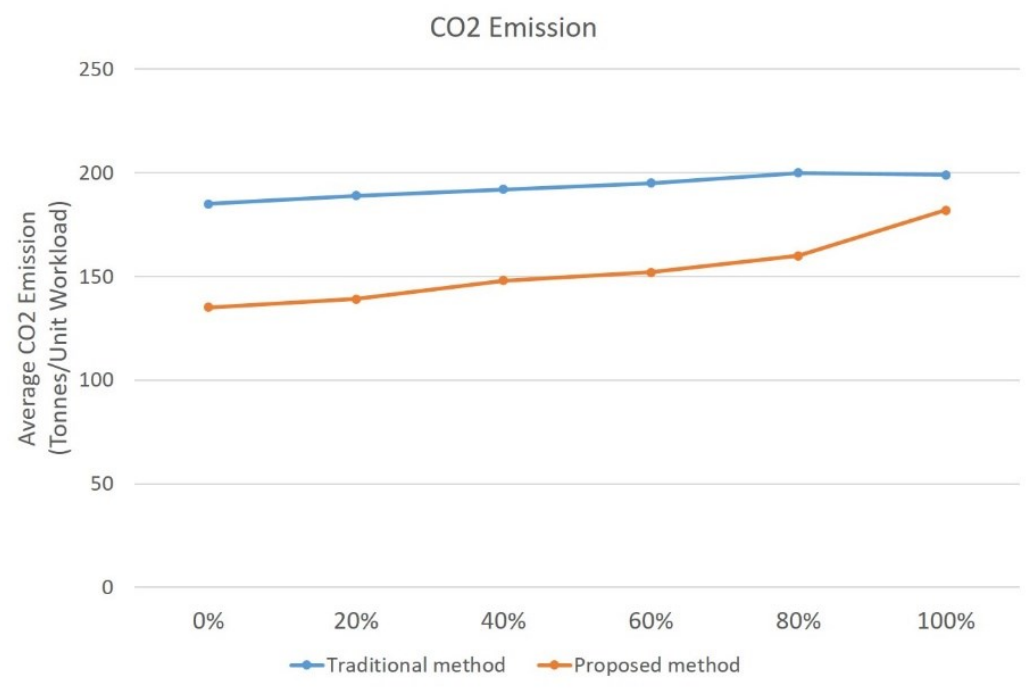

(b)

Figure 3 Performance comparison based on energy consumption and $\mathrm{CO} 2$ emission between traditional method and proposed method of green cloud computing optimization

\section{CONCLUSION AND FUTURE SCOPE}

Sustainable advancements of cloud computing requires application of green computing technologies. The green cloud computing solution presented in this paper addresses operational cost and environmental impact and optimizes the environment. Further optimization of factors such as virtualization, tenancy and consolidation are to be considered in future work. It is essential to enhance the complete lifespan of virtualization procedure. Privacy and security concerns of tenancy are to be addressed. Calculation of threshold value based on multiple aspects, virtual machine consolidation with intelligent support system and management of server downtime are also major challenges that are to be addressed.

\section{References}

[1] Radu, Laura-Diana. "Green cloud computing: A literature survey." Symmetry 9, no. 12 (2017): 295.

[2] Patel, Yashwant Singh, Neetesh Mehrotra, and Swapnil Soner. "Green cloud computing: A review on Green IT areas for cloud computing environment." In 2015 International Conference on Futuristic Trends on Computational Analysis and Knowledge Management (ABLAZE), pp. 327-332. IEEE, 2015. 
[3] Kaushik, Rini T., Prasenjit Sarkar, and Abdullah Gharaibeh. "Greening the compute cloud's pricing plans." In Proceedings of the workshop on power-aware computing and systems, p. 6. ACM, 2013.

[4] Jain, Anubha, Manoj Mishra, Sateesh Kumar Peddoju, and Nitin Jain. "Energy efficient computing-green cloud computing." In 2013 International Conference on Energy Efficient Technologies for Sustainability, pp. 978-982. IEEE, 2013.

[5] Godbole, Nina S., and John Lamb. "The triple challenge for the healthcare industry: sustainability, privacy, and cloud-centric regulatory compliance." In 2013 10th International Conference and Expo on Emerging Technologies for a Smarter World (CEWIT), pp. 1-6. IEEE, 2013.

[6] Pawlish, Michael, Aparna S. Varde, and Stefan A. Robila. "Cloud computing for environment-friendly data centers." In Proceedings of the fourth international workshop on Cloud data management, pp. 43-48. ACM, 2012.

[7] Yamini, B., and D. Vetri Selvi. "Cloud virtualization: A potential way to reduce global warming." In Recent Advances in Space Technology Services and Climate Change 2010 (RSTS \& CC-2010), pp. 55-57. IEEE, 2010.

[8] Gayathri, B. "Green cloud computing." (2012): 114-118.

[9] Dougherty, Brian, Jules White, and Douglas C. Schmidt. "Model-driven auto-scaling of green cloud computing infrastructure." Future Generation Computer Systems 28, no. 2 (2012): 371-378.

[10] Jeba, Jenia Afrin, Shanto Roy, Mahbub Or Rashid, Syeda Tanjila Atik, and Md Whaiduzzaman. "Towards Green Cloud Computing an Algorithmic Approach for Energy Minimization in Cloud Data Centers." International Journal of Cloud Applications and Computing (IJCAC) 9, no. 1 (2019): 59-81.

[11] Borah, Amlan Deep, Deboraj Muchahary, Sandeep Kumar Singh, and Janmoni Borah. "Power saving strategies in green cloud computing systems." International Journal of Grid Distribution Computing 8, no. 1 (2015): 299-306.

[12] Kumar, Kancharana Nithin, and Richa Vasuja. "A Novel Scheme of Computing: Green Cloud Computing." International Journal of Scientific Research in Computer Science, Engineering and Information Technology IJSRCSEIT 3, no. 3 (2018). 
[13] Patil, Archana, and Dr Patil. "An Analysis Report on Green Cloud Computing Current Trends and Future Research Challenges." An Analysis Report on Green Cloud Computing Current Trends and Future Research Challenges (March 19, 2019) (2019).

[14] Haghighi, Maryam Askarizade, Mehrdad Maeen, and Majid Haghparast. "An Energy-Efficient Dynamic Resource Management Approach Based on Clustering and Meta-Heuristic Algorithms in Cloud Computing IaaS Platforms." Wireless Personal Communications 104, no. 4 (2019): 1367-1391.

[15] Garg, Saurabh Kumar, Chee Shin Yeo, and Rajkumar Buyya. "Green cloud framework for improving carbon efficiency of clouds." In European Conference on Parallel Processing, pp. 491-502. Springer, Berlin, Heidelberg, 2011.

[16] Satoh, Fumiko, Hiroki Yanagisawa, Hitomi Takahashi, and Takayuki Kushida. "Total energy management system for cloud computing." In 2013 IEEE International Conference on Cloud Engineering (IC2E), pp. 233 240. IEEE, 2013.

[17] Patil, Archana, and Dr Patil. "An Analysis Report on Green Cloud Computing Current Trends and Future Research Challenges." An Analysis Report on Green Cloud Computing Current Trends and Future Research Challenges (March 19, 2019) (2019).

[18] Jing, Si-Yuan, Shahzad Ali, Kun She, and Yi Zhong. "State-of-the-art research study for green cloud computing." The Journal of Supercomputing 65, no. 1 (2013): 445-468.

[19] Buyya, Rajkumar, Anton Beloglazov, and Jemal Abawajy. "Energy-efficient management of data center resources for cloud computing: a vision, architectural elements, and open challenges." arXiv preprint arXiv:1006.0308 (2010).

[20] Pazowski, Piotr. "Green computing: latest practices and technologies for ICT sustainability." In Managing Intellectual Capital and Innovation for Sustainable and Inclusive Society, Joint International Conference, Bari, Italy, pp. 1853-1860. 2015. 